Relations industrielles

Industrial Relations

\title{
Labor Economics, by R.B. Freeman, Englewood Cliffs, N.J., Prentice Hall, 1972, 136 pp.
}

\section{Gérard Bélanger}

Volume 27, numéro 4, 1972

URI : https://id.erudit.org/iderudit/028341ar

DOI : https://doi.org/10.7202/028341ar

Aller au sommaire du numéro

Éditeur(s)

Département des relations industrielles de l'Université Laval

ISSN

0034-379X (imprimé)

1703-8138 (numérique)

Découvrir la revue

Citer ce compte rendu

Bélanger, G. (1972). Compte rendu de [Labor Economics, by R.B. Freeman, Englewood Cliffs, N.J., Prentice Hall, 1972, 136 pp.] Relations industrielles / Industrial Relations, 27(4), 785-786. https://doi.org/10.7202/028341ar

Tous droits réservés (C Département des relations industrielles de l'Université Laval, 1972
Ce document est protégé par la loi sur le droit d'auteur. L'utilisation des services d'Érudit (y compris la reproduction) est assujettie à sa politique d'utilisation que vous pouvez consulter en ligne.

https://apropos.erudit.org/fr/usagers/politique-dutilisation/ 
suggestion à l'effet que dans les cas où le gouvernement jugerait bon de ne pas suggérer l'approbation des crédits nécessaires à l'application de la sentence arbitrale, celui-ci aurait à motiver ses raisons devant la législature qui, ultimement, possède l'autorité d'accorder ces crédits. En effet, en démocratie parlementaire britannique, le gouvernement contrôle la législature par sa majorité, et l'on conçoit difficilement que l'opposition puisse convaincre celui-ci d'accorder les crédits. Il s'agit là, selon nous, d'une des limites à la négociation collective dans le secteur public et c'est ce qui rend l'institution différente de celle du secteur privé.

Une autre recommandation empruntée au modèle fédéral touche la détermination des services essentiels dans les cas où les syndiqués auraient choisi l'option droit de grève. Comme au fédéral, la Commission suggère la désignation d'employés ou classes d'employés dits « essentiels $\gg$ et qui devront continuer à travailler dans l'éventualité d'une grève. La liste de ces employés est également établie de la même façon qu'au fédéral : énumération par l'employeur, contestation par le syndicat s'il y a lieu, et arbitrage par la Commission des relations du travail.

Enfin, dans le domaine de l'action politique, la Commission Cohen interdit aux seuls fonctionnaires de faire de la politique partisane. Elle leur permet cependant de s'affilier à n'importe quelle centrale à la condition que celle-ci ne force pas les fonctionnaires à contribuer financièrement à un parti politique.

La cinquième partie du rapport suggère les changements appropriés dans l'administration de la législation en vue de mettre en pratique les recommandations contenues dans les trois parties précédentes. Ainsi, on recommande que la Commission des relations du travail possède un président et autant de viceprésidents qu'il semblera bon d'y en avoir à plein temps. Que cette Commission soit formée de représentants d'employeurs et d'associations d'employés plutôt que composée d'experts. Qu'elle soit complètement indépendante du gouvernement. Que ce soit la même qui ait juridiction sur le secteur public en y ajoutant le personnel nécessaire et qu'elle fonctionne en comités paritaires : un pour le secteur public, un pour l'industrie des pêcheries, un pour l'industrie de la construction et un pour toutes les autres industries.
Les décisions de la Commission des relations du travail touchant les pratiques interdites ainsi que les grèves et lockouts illégaux devraient avoir force de loi comme les décisions de la Cour suprême de Terre-Neuve, selon la Commission d'enquête.

Le rapport Cohen recommande également la création d'une Commission de revision ( Fair Representation Review Board $\gg)$ composée d'une ou plusieurs personnes neutres pour entendre les plaintes des individus envers leur syndicat. Enfin, on suggère que le personnel du Ministère du travail soit augmenté de façon à établir des conseils régionaux tripartites (un représentant des employeurs, un représentant des employés et un président impartial choisi parmi des personnes jugées dignes de confiance dans une région donnée) dont les fonctions seraient : faire respecter les diverses lois du travail (salaire minimum, accidents du travail, etc.); faire des recommandations pour améliorer ces lois ; s'occuper de toutes autres questions pouvant faire l'objet d'une politique gouvernementale concernant les normes du travail. L'idée majeure derrière ces conseils régionaux est de protéger le plus adéquatement possible les travailleurs non organisés. Un bureau de recherche sur les salaires des secteurs public et privé devrait aussi être établi.

La seule critique que nous pouvons formuler à l'endroit du rapport Cohen est qu'il recommande trop de tâches concentrées dans la seule personne du président de la Commission des relations du travail. Nous croyons que ce président sera trop accaparé pour remplir efficacement chacune des fonctions qu'on lui aura attribuées. Dans l'ensemble, cependant, il s'agit là d'un travail excellent dont plusieurs autres législations canadiennes auraient intérêt à s'inspirer pour améliorer leurs législations désuètes dans le domaine des relations du travail.

\section{Jean BOIVIN}

Labor Economics, by R.B. Freeman, Englewood Cliffs, N.J., Prentice Hall, 1972, 136 pp.

Dernier venu de l'excellente collection Foundations of Modern Economic Series, ce manuel d'économique du travail possède les qualités des publications antérieures : excellente vulgarisation du sujet dans une présentation soignée. Le volume 
se divise en deux parties: les cinq premiers chapitres sont d'ordre analytique et appliquent les outils conventionnels de l'analyse de marché à l'étude des différentes facettes du facteur de production, travail. Les deux derniers chapitres initient le lecteur aux problèmes des relations industrielles proprement dites.

En traitant de multiples aspects de façon concise, l'auteur soutient du début à la fin l'intérêt du lecteur. Ce livre a cependant le défaut de ses qualités. Comme la majorité des manuels introductifs, ce livre tend à donner la fausse impression de solutionner les problèmes au lieu de laisser le lecteur sur des interrogations qui l'inciteraient à poursuivre une réflexion. Il y a cependant une exception, lors de la discussion des courbes Phillips et des attentes des agents économiques sur le niveau des prix futurs. Ici l'auteur insiste sur l'existence d'une controverse sur le sujet et la nécessité de meilleurs travaux théoriques et empiriques. Ecrit avant août 1972, ce livre n'aborde pas une question d'actualité, soit les implications des différentes politiques de revenu.

En résumé, un excellent manuel introductif à l'économique du travail.

\section{Gérard BELANGER}

\section{The Canadian Labour Market, Readings} in Manpower Economics, by Arthur Kruger and Noah M. Meltz, Toronto, Centre for Industrial Relations, University of Toronto, 1968, 312 pp.

The Canadian Labour Market est un livre de lectures choisies. Mais, à l'encontre de la plupart des livres de ce genre, les textes n'ont pas été extraits de livres ou de revues; ils ont été préparés expressément pour être publiés dans ce livre. Les études ont donc le grand avantage d'être originales et de très bien s'intégrer les unes aux autres pour former un ensemble cohérent. De plus, le choix des sujets a été fait de façon très judicieuse, en ce sens que chaque sujet revêt une importance très grande dans le marché du travail canadien. Chaque sujet sera d'ailleurs recensé brièvement dans le texte qui suit, ceci dans l'ordre où chacun apparaît dans le livre.

Micro-economic Theory, Labour Allocation and Manpower Policy, par Arthur Kruger.
La fonction primordiale du marché du travail, comme de tout marché économique d'ailleurs, consiste à allouer les travailleurs aux différents emplois dans l'économie. Dans l'économie de marché canadienne, cette allocation se fait principalement par le mécanisme des prix, le prix du travail s'appelant salaire. Kruger a donc raison d'expliquer, dans ce texte, comment l'économique, et en particulier la micro-économique, facilite la compréhension de cette allocation du travail. L'auteur présente de façon simple et en même temps claire le rôle de l'offre et de la demande du travail, dans la détermination des salaires et des niveaux d'emploi dans les divers marchés du travail. Il souligne cependant deux imperfections, parmi d'autres, qui empêchent les marchés du travail de fonctionner tel que le prédit la théorie économique. $\mathrm{La}$ première imperfection provient du pouvoir des parties en présence; l'autre de la mobilité imparfaite des travailleurs. Tout au long de son texte, l'auteur suggère des politiques de main-d'œuvre susceptibles d'améliorer le fonctionnement des marchés du travail. Finalement, le lecteur moins familier avec la micro-économique peut lire avec profit quelques explications sur les principaux concepts de base dans cette partie de l'économique.

The Analysis of the Labour Market in Classical Economics, par Samuel Hollander.

On sait que la plupart des économistes, dits classiques, qui ont étudié le marché du travail l'ont fait en grande partie au niveau de toute l'économie, à partir de Smith (1776) à Cairnes (1874). Ils se sont donc intéressés à la détermination du taux moyen de salaire et au niveau de l'emploi total. Il faut de plus être conscient du fait que la principale théorie classique des salaires se rapportait surtout à l'offre du travail; il s'agit de la théorie de la subsistance. L'auteur en explique l'essentiel. Il fait de même d'une autre théorie classique qui, elle, se situe du côté de la demande, i.e. la théorie du fonds de salaire. Dans la suite, l'auteur essaie d'évaluer la validité de ces deux théories classiques. Puis, il explique les vues des classiques sur les trois questions suivantes : le chômage; la structure des salaires et le rôle du gouvernement dans l'éducation; et finalement leur atti- 